\title{
Candida central nervous system infection after neurosurgery: a single-institution case series and literature review
}

\author{
Hongwei Chen $^{1 \#}$, Wenkai Cong ${ }^{1 \#}$, Dongcheng Xie ${ }^{1}$, Shengjie Wang ${ }^{1}$, Jianxing Niu ${ }^{1}$, Guoqiang Chen ${ }^{2}$ \\ Xuanwei Dong ${ }^{1}$, Qingming Zhou ${ }^{2}$ \\ ${ }^{1}$ Department of Neurosurgery for Cerebrospinal Fluid Diseases, Aviation General Hospital, Beijing, China; ${ }^{2}$ Department of Neurosurgery, Aviation \\ General Hospital, Beijing, China \\ Contributions: (I) Conception and design: H Chen, Q Zhou; (II) Administrative support: G Chen; (III) Provision of study materials or patients: H \\ Chen; (IV) Collection and assembly of data: W Cong, D Xie, S Wang, J Niu; (V) Data analysis and interpretation: H Chen, W Cong, X Dong; (VI) \\ Manuscript writing: All authors; (VII) Final approval of manuscript: All authors. \\ \#These authors contributed equally to this work and are co-first authors. \\ Correspondence to: Qingming Zhou. Department of Neurosurgery, Aviation General Hospital, No. 3 Anwai Beiyuan, Chaoyang District, Beijing \\ 100012, China. Email: 346663509@qq.com.
}

Background: Candida central nervous system (CNS) infection is a rare complication following
neurosurgery. This disease often occurs during the treatment of bacterial CNS infection, and common
bacterial culture results have a high false negative rate, which delays diagnosis and treatment, and seriously
affect the prognosis of patients. The purpose of this study was to discuss the diagnosis, treatment process,
and results of this disease through a small series of cases, so as to provide data support and a theoretical basis
for the timely diagnosis and treatment of Candida CNS infection after neurosurgery. Methods: A retrospective analysis was conducted on eight patients with confirmed Candida CNS infection following neurosurgery in our department between June 2011 and June 2019. Their clinical symptoms, treatment schemes, outcomes, risk factors, and complications were analyzed.

Results: Four patients received intravenous administration of fluconazole and were cured. Three patients received intravenous administration of amphotericin B. Two of them were cured, and the other died. One patient was cured after intravenous administration of voriconazole throughout the treatment. The overall cure rate was $87.5 \%(7 / 8)$, and the death rate was $12.5 \%(1 / 8)$. Among the three patients treated by amphotericin B, one patient suffered vomiting and renal function impairment. After drug discontinuation, this patient gradually improved. Another patient had acute renal failure, and the conditions were not improved after drug discontinuation. The remaining patient suffered from anemia and vomiting, which were relieved after drug discontinuation. One patient had hematuria during voriconazole treatment, and the symptoms were improved after the therapy was changed to fluconazole. Four patients treated with fluconazole did not have apparent adverse reactions. None of the cured patients relapsed during the 3-12 months follow-up after discharge.

Conclusions: Candida CNS infection following neurosurgery is a rare condition; however, it may result in disastrous consequences. Early diagnosis and timely use of antifungal agents are considered the primary treatment principles. Blood culture of cerebrospinal fluid (CSF) is useful for early diagnosis. Fluconazole is the preferred choice for the clinical treatment of Candida CNS infection as it has both good efficacy and safety.

Keywords! Candida; central nervous system infection; neurosurgery; fluconazole; case series

Submitted May 14, 2021. Accepted for publication Oct 29, 2021.

doi: 10.21037/apm-21-2577

View this article at: https://dx.doi.org/10.21037/apm-21-2577 


\section{Introduction}

Postoperative central nervous system (CNS) infection following neurosurgery is a complication characterized by high disability and mortality (1), with the incidence of $1-10 \%$ (2). Bacterial infections are the most common type of postoperative CNS infection following neurosurgery, especially Gram-positive cocci, Staphylococcus epidermidis, and Staphylococcus aureus infections. Cerebrospinal fluid (CSF) leakage and foreign body implantation may be the main causes of CNS infection after neurosurgery $(3,4)$. Fungal CNS infections, such as Candida, have a relatively low incidence (5). Candida CNS infection shares similar symptoms with bacterial CNS infection, such as fever, nuchal rigidity, altered mental status, and headache (6). Both may present as meningitis or related symptoms, which are easily neglected clinically, thereby resulting diagnostic and treatment delays and affecting the prognosis, and even leading to death (7). Low immunity, broad-spectrum antibiotics and anemia may be the risk factors of Candida CNS infection (3). Neurosurgery will destroy the bloodbrain barrier and damage the natural defense mechanism of human body. Candida may increase in value and invade the subarachnoid space and brain (8).

In the present study, medical records were collected from patients with Candida CNS infection after neurosurgery at the Cerebrospinal Fluid (CSF) Disease Center of Aviation General Hospital. Their clinical features and risk factors were analyzed, and a particular emphasis was placed on our diagnostic and treatment methods for these patients. Our research findings will provide reference for the diagnosis and treatment of similar patients. We present the following article in accordance with the AME Case Series reporting checklist (available at https://dx.doi.org/10.21037/apm-21-2577).

\section{Methods}

\section{General data}

From June 2011 to June 2019, eight patients with confirmed postoperative Candida CNS infection following neurosurgery at the CSF Diagnosis and Treatment Center of Aviation General Hospital of China Medical University were recruited. All patients had symptoms of CNS infection. Candida infection was confirmed in all of these patients by CSF culture, either by using a blood culture bottle or routine procedures (Table 1). We reviewed the basic information of each patient, including age, gender, primary disease, history of Neurosurgery, history of previous bacterial CNS infection, history of antibiotic application, etc. Clinical data and results of antifungal therapy in each patient were collected. Follow up was conducted by outpatient and telephone. The patients were followed up for 3-12 months. Three of the eight patients were first confirmed as positive for Candida species by CSF culture in a blood culture bottle; however, they were initially negative for CSF culture. Subsequently, blood or CSF cultures were performed repeatedly, and all were positive. One patient was initially found to be positive for Candida species by CSF culture in a blood culture bottle, while the CSF culture was consistently negative. Two patients were simultaneously found to be positive for Candida species by CSF culture in a blood culture bottle and by ordinary CSF culture. Another two patients were confirmed as positive for Candida infection only through ordinary CSF culture.

The patients comprised seven males and one female, aged $1-52$ years old, with an average of $30.1 \pm 16.6$ years. Primary diseases among these patients included the following: (I) traumatic brain injury in two patients; (II) subarachnoid hemorrhage in one patient; (III) intracranial tumor in two patients; (IV) hydrocephalus in two patients; and $(\mathrm{V})$ otitis media-induced brain abscess in one patient. All patients were surgically treated for primary diseases. Seven patients had bacterial CNS infections combined with hydrocephalus, as well as a recent history of antibiotics use and a ventriculoperitoneal shunt (VPS) or external ventricular drain (EVD).

The clinical manifestations upon confirmation of Candida infection were as follows: loss of consciousness in three patients, drowsiness in two patients, and clear consciousness in three patients. There were seven patients with fever (body temperature $>38{ }^{\circ} \mathrm{C}$ ), one patient with normal body temperature, and eight patients positive for meningeal irritation syndrome. This study has been approved by the Medical Ethics Committee of Aviation General Hospital (2021-KY-01-11). The study was a retrospective study and the data was managed anonymously, so the requirement for informed consent was waived. We have a policy on patient privacy. The study was carried out in accordance with the Declaration of Helsinki (as revised in 2013).

\section{Treatment}

Four patients underwent removal of the VPS at an early stage following admission (less than $72 \mathrm{~h}$ ), and received EVD. Three patients carried EVDs upon admission, and the drains were unobstructed and retained. One of these 


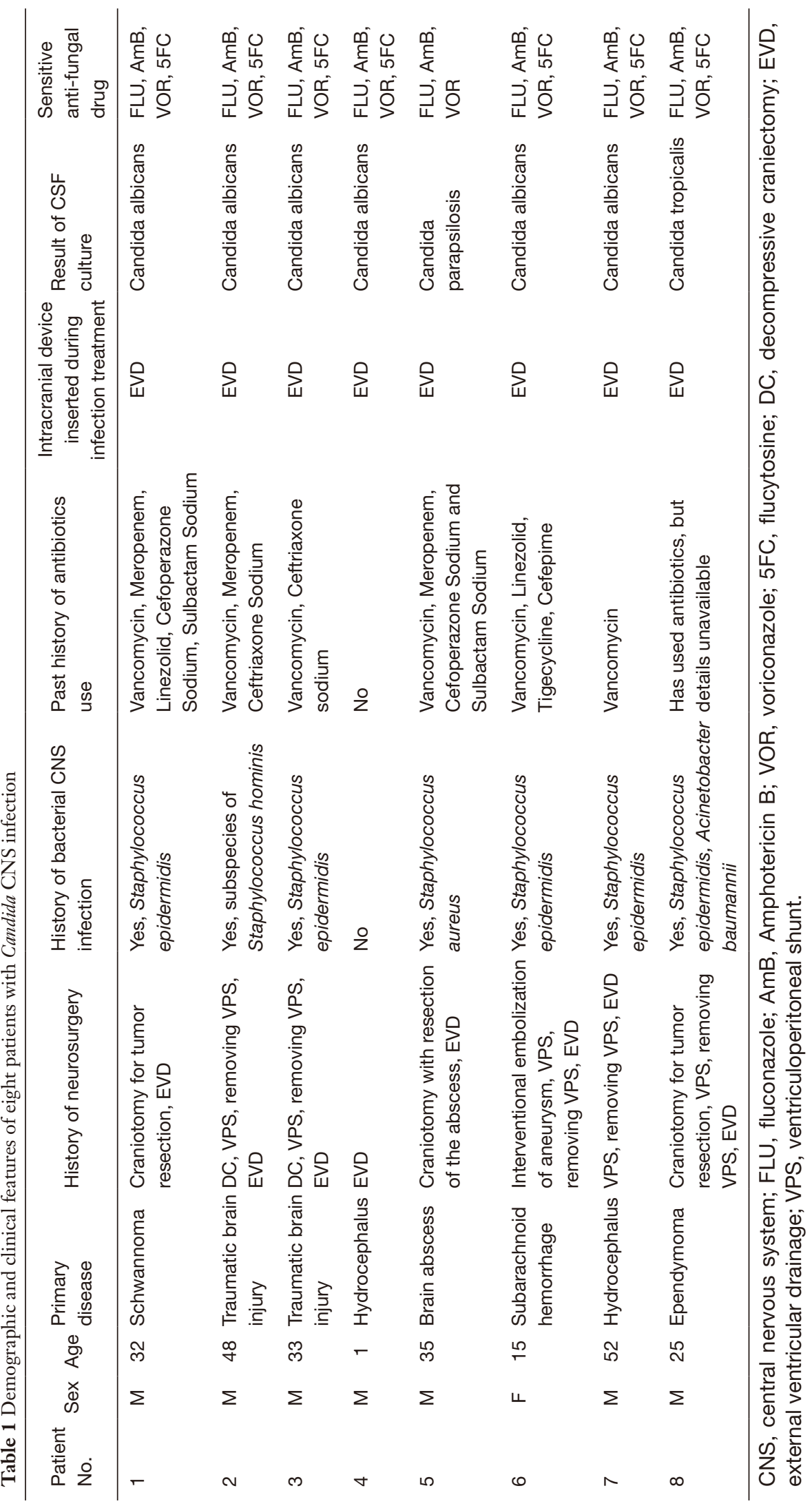


Table 2 Treatment schemes and results of eight patients with Candida CNS infection

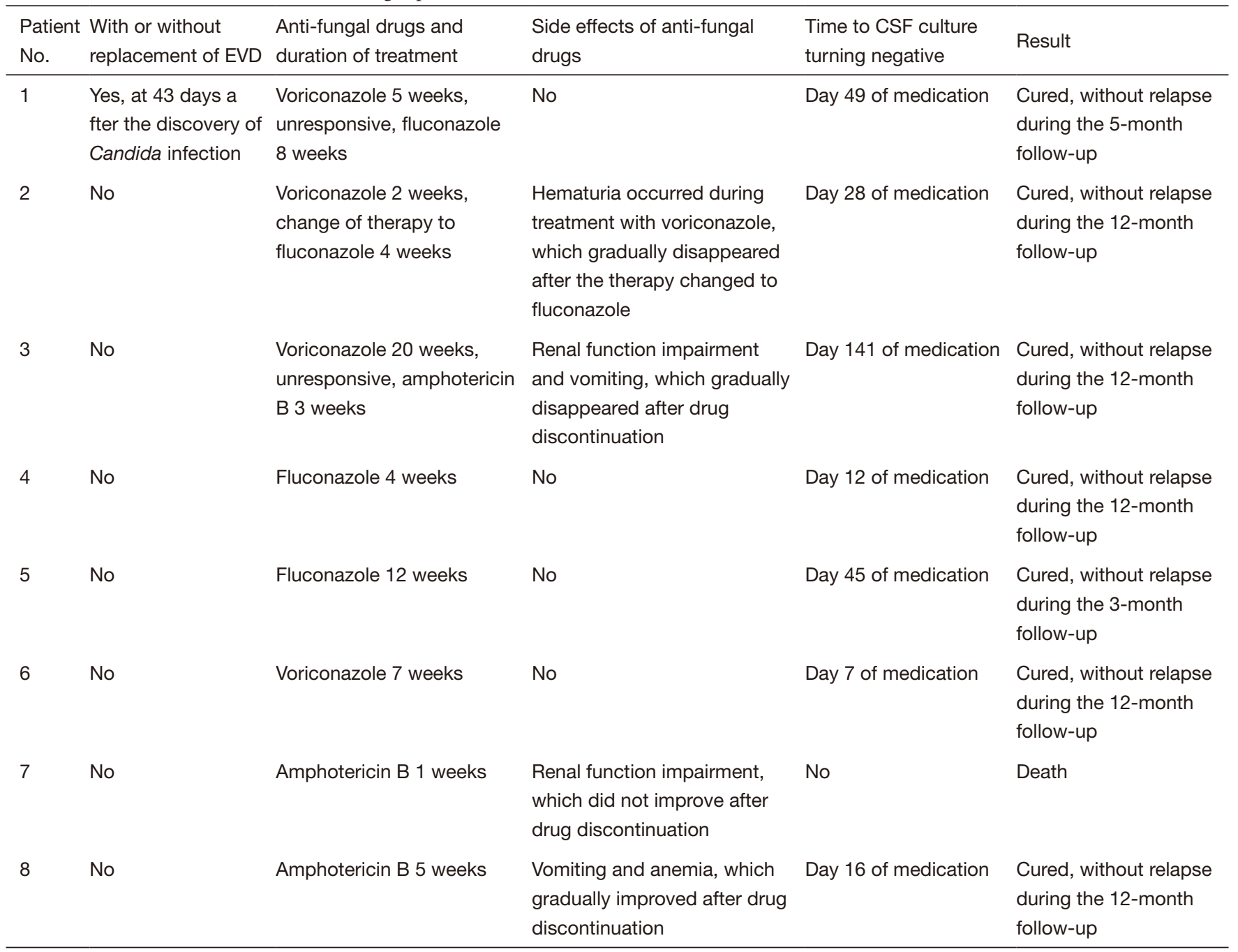

CNS, central nervous system; EVD, external ventricular drainage; CSF, cerebrospinal fluid.

three patients had an obstructed drain replaced during infection treatment. One patient with hydrocephalus directly received EVD upon admission. Following confirmation of the diagnosis, anti-fungal drugs were given by intravenous administration according to the drug allergy information in Table 1. The treatment schemes for each patient are shown in Table 2. None of these patients received an intraventricular injection of anti-fungal drugs. All seven patients, who were cured after CNS infection, received VPS.

\section{Observation indicators}

Changes in symptoms and signs before and after treatment were observed and recorded, as was the duration of medication use. CSF samples were taken and preserved regularly each week for culturing (ordinary CSF culture and CSF culture in a blood culture bottle). CSF biochemistry and other routine tests were also performed. Other tests included routine blood tests, liver and kidney function tests, and electrolyte tests.

\section{Efficacy evaluation}

According to the Clinical Practice Guideline for the Management of Candidiasis: 2016 Update by the Infectious Diseases Society of America (9), cure was defined as the 
disappearance of symptoms, signs, and CSF and radiologic abnormalities related to Candida CNS infection.

\section{Safety evaluation}

Adverse events related to medication were closely observed, including renal and hepatic toxicity, electrolyte disturbance, anemia, leukopenia, thrombocytopenia, nausea, vomiting, diarrhea, and convulsions. The incidence of adverse events was estimated.

\section{Statistical analysis}

Continuous variables were reported as the median and range, while categorical variables were reported as frequencies and percentages. Data analysis was performed in Excel 16.0 (Microsoft Corporation, USA).

\section{Results}

\section{Clinical efficacy}

Four patients received intravenous administration of fluconazole and were cured. One of them was initially treated by voriconazole but was unresponsive, and was later shifted to fluconazole. Another was initially treated by voriconazole and was later shifted to fluconazole due to hematuria. Two patients were cured after treatment with amphotericin B. One of these patients was initially treated by voriconazole but was unresponsive, and was later shifted to amphotericin $\mathrm{B}$, while the other patient died. One patient was cured following intravenous administration of voriconazole throughout the treatment (Table 2). The overall cure rate was $87.5 \%(7 / 8)$, with one withdrawal (death) (Table 2). The seven cured patients received anti-fungal treatment for 1-23 weeks, with an average of $10.0 \pm 6.7$ weeks. Following treatment, the results of several consecutive CSF cultures (over three times) were negative, with the disappearance of related symptoms, signs, and radiological abnormalities. The relatives of one patient abandoned treatment, and this patient died soon after discharge. The cause of death of this patient was an extensive infection of brain parenchyma with acute renal failure.

\section{Safety evaluation}

During infection treatment, one out of three patients treated with amphotericin B presented with renal function impairment and vomiting. The conditions were relieved after drug discontinuation. One patient suffered acute renal failure, and the conditions were not improved after drug discontinuation. The remaining patient had anemia and vomiting, which were gradually improved after drug discontinuation. Hematuria occurred in one patient during voriconazole treatment, which disappeared after the therapy was changed to fluconazole. Four patients, who were treated by fluconazole, did not have apparent adverse reactions during treatment.

\section{Long-term follow-up}

The seven cured patients were followed up for 3-12 months after discharge, with an average of $9.7 \pm 3.9$ months, and none of them relapsed.

\section{Discussion}

Candida CNS infection following neurosurgery is rare $(5,10,11)$. Nguyen et al. only reported three such cases at their institute during the 4-year period from 1989 to 1993 (11). Nearly 100 patients with conditions associated with intracranial infections across China visit our center every year. Over a period of 8 years, there were only eight patients with Candida CNS infection, which only accounted for approximately $1.00 \%$ of all intracranial infections. Candida albicans is the most common cause of postoperative Candida CNS infection following neurosurgery $(10,12)$, which is consistent with our statistics (Table 1). Previous reports have shown that the mortality of Candida CNS infection ranges from approximately $10-33 \%$ (13), which also in agreement with our data (12.5\%). Patients with postoperative Candida CNS infection following neurosurgery usually have recent history of bacterial CNS infection, antibiotics use, and CNS surgery (14). O'Brien et al. performed a retrospective analysis that showed that the risk factors for postoperative Candida CNS infection following neurosurgery include external intracranial devices, bacterial CNS infection, as well as the use of wide-spectrum antibiotics (10). In the present study, the risk factors identified in the eight included patients were similar to those in previous reports. All patients in our study had a recent history of CNS surgery, and seven of them had a recent history of bacterial CNS infection and antibiotics use.

Although ordinary CSF culture can identify pathogens, the false negative rate remains high clinically (7). Possible reasons for this include the following: (I) the volume 
of the CSF sample used for the culture is small or the content of pathogens in the sample is low; (II) pathogens in the CSF sample have a high requirement on the growth environment; and (III) patients have previously been prescribed antifungal agents prior to identification of the pathogens. Simor et al. showed that the blood culture system could improve the pathogen detection rate in bodily fluids (other than blood) and shorten the time needed for detection. This is because the blood culture flask usually contains the growth supplements, activated charcoal, and Fuller's earth that are conducive to the detection of pathogens in CSF (15). According to Yoo et al., the positive rate of CSF culture in a blood culture bottle is significantly higher than that of ordinary CSF culture (16). Four patients in the present study were initially confirmed as positive for Candida species by CSF culture in a blood culture bottle; however, they were negative for ordinary CSF culture at this time. Subsequently, three of them were found to be positive by repeated CSF cultures in a blood culture bottle as well as ordinary CSF culture, and one of them was constantly negative for the ordinary CSF culture. This indicates that for symptomatic patients, CSF culture should be performed in a blood culture bottle in order to improve the positive rate, rapidly identify pathogens, and select the appropriate medication.

For the treatment of Candida CNS infection, intravenous administration of amphotericin B plus flucytosine is usually recommended (9). Amphotericin B is a polyene antifungal drug, which inhibits fungal growth by affecting cell membrane permeability. It is active against all Candida species, and there is a large body of evidence supporting the use of amphotericin B for the treatment of Candida CNS infection (17). However, amphotericin B is associated with greater toxicity, with renal toxicity being the most common (18). In this study, two of the three patients treated with amphotericin B suffered renal function impairment. Of these patients, one was a mild case whose condition gradually improved after drug discontinuation. However, the other patient suffered acute renal failure during treatment with this medication, and their condition did not improve after drug discontinuation. This patient's relatives refused continuous renal replacement therapy (CRRT), which might have been an important factor that leads to the patient's death.

CSF is highly permeable to flucytosine, which is active against most fungi in the clinic. However, its high cost and toxicity have restricted its application (19). Voriconazole is a second-generation triazole anti-fungal drug, which displays excellent in vitro anti-fungal activity against a variety of yeasts and molds. Voriconazole has a more potent anti-fungal spectrum than the first-generation, but also involves more side effects and drug-drug interactions than fluconazole. There is also a lack of clinical trial data supporting the use of voriconazole in Candida CNS infections (20). In our study, four patients were initially treated with voriconazole, and another patient was consistently treated with voriconazole throughout the course of the disease. Two patients were unresponsive to at least 2 weeks of voriconazole treatment and were shifted to fluconazole or amphotericin B. These two cases were eventually cured. Hematuria occurred in one patient during voriconazole treatment, and the symptoms disappeared after the therapy changed to fluconazole. Voriconazole is a triazole anti-fungal drug, to which the CNS is highly permeable. It is active against most strains of Candida causing CNS infections (9), in addition to having a high tolerance in most patients (18). In this study, four patients were cured after treatment with fluconazole and did not present with adverse events. This indicated that fluconazole outperformed voriconazole in efficacy and had a better safety profile than amphotericin B. At present, there are no guidelines available concerning the duration of anti-fungal treatment for Candida CNS infections. As for the duration of treatment for candidemia, guidelines recommend continued use for 2 weeks after the blood culture turns negative and the symptoms and signs of candidemia disappear (9). The guidelines for candidemia were referred to when determining the duration of treatment for Candida CNS infection. That is, the medication was continued for at least 2 weeks after the CSF culture turned negative, routine CSF test and biochemistry indicators were normal, and symptoms of infection disappeared. In the present study, the CSF culture turned negative in 7-141 days (Table 2), and the median treatment time was 28 days, which was close to the reported median treatment time of 28 days (14-41 days) in the previous studies (11). Moreover, no recurrence of infection was observed during the long-term follow-up.

Studies have shown that Candida infection causes the formation of biofilm over the medical device, which adds to the difficulty of eliminating the pathogens by medication alone, thereby increasing the risk of recurrent infection $(10,21,22)$. We believe that as long as CNS drainage is unobstructed, it is not necessary to remove the EVD at an early stage, due to the following considerations. Firstly, the EVD is a replaceable device that can drain the CSF with fungal colonization outside the body, thus reducing 
secondary injury to the brain tissues and nervous system. Secondly, the EVD provides an intuitive pathway to observe the properties of the CSF, based on which the status of the intracranial infection can be assessed regularly. EVD plays a vital role in the treatment of Candida CNS infection. In contrast, the VPS has little clinical value and should be removed as early as possible. A study reported that continuous EVD was beneficial for the treatment of fungal CNS infections (23). In this study, the EVD was not removed at an early stage in three patients, but was removed only after healing of the infection and during the subsequent VPS. All of these patients were cured and did not present with complications. All cured patients were combined with hydrocephalus and received VPS after the healing of the CNS infection. These patients were followed up for 3-12 months after discharge, and there was no relapse of CNS infections or hydrocephalus.

There are some limitations in this study that should be noted. Firstly, this was a single-center and retrospective study that had a limited sample size. We will further conduct multi-center and large sample research. Secondly, intrathecal or intracerebroventricular administration of anti-fungal drugs was performed in the present study. Although some successful cases have been reported $(24,25)$, the working mechanism of this administration pathway in the treatment of Candida CNS infection is not yet fully understood.

\section{Conclusions}

Candida CNS infection following neurosurgery is a rare condition; however, it may result in disastrous consequences. Early diagnosis and timely use of antifungal agents are considered the primary treatment principles. Blood culture of CSF is useful for early diagnosis. Fluconazole is the preferred choice for the clinical treatment of Candida CNS infection as it has good efficacy and safety. Internal and external ventricular drains can be temporarily retained during infection treatment.

\section{Acknowledgments}

Funding: None.

\section{Footnote}

Reporting Checklist: The authors have completed the AME Case Series reporting checklist. Available at https://dx.doi. org/10.21037/apm-21-2577
Data Sharing Statement: Available at https://dx.doi. org/10.21037/apm-21-2577

Conflicts of Interest: All authors have completed the ICMJE uniform disclosure form (available at https://dx.doi. org/10.21037/apm-21-2577). The authors have no conflicts of interest to declare.

Ethical Statement: The authors are accountable for all aspects of the work in ensuring that questions related to the accuracy or integrity of any part of the work are appropriately investigated and resolved. This study has been approved by the Medical Ethics Committee of Aviation General Hospital (2021-KY-01-11). The study was a retrospective study and the data was managed anonymously, so the requirement for informed consent was waived. The study was carried out in accordance with the Declaration of Helsinki (as revised in 2013).

Open Access Statement: This is an Open Access article distributed in accordance with the Creative Commons Attribution-NonCommercial-NoDerivs 4.0 International License (CC BY-NC-ND 4.0), which permits the noncommercial replication and distribution of the article with the strict proviso that no changes or edits are made and the original work is properly cited (including links to both the formal publication through the relevant DOI and the license). See: https://creativecommons.org/licenses/by-nc-nd/4.0/.

\section{References}

1. Golan E, Wong K, Alahmadi H, et al. Endoscopic sphenoid sinus drainage in Lemierre syndrome. J Clin Neurosci 2014;21:346-8.

2. Reichert MC, Medeiros EA, Ferraz FA. Hospitalacquired meningitis in patients undergoing craniotomy: incidence, evolution, and risk factors. Am J Infect Control 2002;30:158-64.

3. Chen M, Chen C, Yang Q, et al. Candida meningitis in neurosurgical patients: a single-institute study of nine cases over 7 years. Epidemiol Infect 2020;148:e148.

4. Korinek AM, Baugnon T, Golmard JL, et al. Risk factors for adult nosocomial meningitis after craniotomy: role of antibiotic prophylaxis. Neurosurgery 2006;59:126-33.

5. McClelland S 3rd, Hall WA. Postoperative central nervous system infection: incidence and associated factors in 2111 neurosurgical procedures. Clin Infect Dis 2007;45:55-9.

6. de Gans J, van de Beek D; European Dexamethasone 
in Adulthood Bacterial Meningitis Study Investigators. Dexamethasone in adults with bacterial meningitis. $\mathrm{N}$ Engl J Med 2002;347:1549-56.

7. Sánchez-Portocarrero J, Pérez-Cecilia E, Corral O, et al. The central nervous system and infection by Candida species. Diagn Microbiol Infect Dis 2000;37:169-79.

8. Góralska K, Blaszkowska J, Dzikowiec M. Neuroinfections caused by fungi. Infection 2018;46:443-59.

9. Pappas PG, Kauffman CA, Andes DR, et al. Clinical Practice Guideline for the Management of Candidiasis: 2016 Update by the Infectious Diseases Society of America. Clin Infect Dis 2016;62:e1-50.

10. O'Brien D, Stevens NT, Lim CH, et al. Candida infection of the central nervous system following neurosurgery: a 12-year review. Acta Neurochir (Wien) 2011;153:1347-50.

11. Nguyen MH, Yu VL. Meningitis caused by Candida species: an emerging problem in neurosurgical patients. Clin Infect Dis 1995;21:323-7.

12. Geers TA, Gordon SM. Clinical significance of Candida species isolated from cerebrospinal fluid following neurosurgery. Clin Infect Dis 1999;28:1139-47.

13. Bridges KJ, Li R, Fleseriu M, et al. Candida Meningitis After Transsphenoidal Surgery: A Single-Institution Case Series and Literature Review. World Neurosurg 2017;108:41-9.

14. Lipton SA, Hickey WF, Morris JH, et al. Candidal infection in the central nervous system. Am J Med 1984;76:101-8.

15. Simor AE, Scythes K, Meaney H, et al. Evaluation of the BacT/Alert microbial detection system with FAN aerobic and FAN anaerobic bottles for culturing normally sterile body fluids other than blood. Diagn Microbiol Infect Dis 2000;37:5-9.

16. Yoo IY, Chun S, Song DJ, et al. Comparison of BacT/

Cite this article as: Chen H, Cong W, Xie D, Wang S, Niu J, Chen G, Dong X, Zhou Q. Candida central nervous system infection after neurosurgery: a single-institution case series and literature review. Ann Palliat Med 2021;10(11):1136211369. doi: 10.21037/apm-21-2577
Alert FAN and FAN Plus Bottles with Conventional Medium for Culturing Cerebrospinal Fluid. J Clin Microbiol 2016;54:2837-40.

17. Kethireddy S, Andes D. CNS pharmacokinetics of antifungal agents. Expert Opin Drug Metab Toxicol 2007;3:573-81.

18. Nivoix Y, Ledoux MP, Herbrecht R. Antifungal Therapy: New and Evolving Therapies. Semin Respir Crit Care Med 2020;41:158-74.

19. Ngan NTT, Mai NTH, Tung NLN, et al. A randomized open label trial of tamoxifen combined with amphotericin $\mathrm{B}$ and fluconazole for cryptococcal meningitis. Wellcome Open Res 2019;4:8.

20. Johnson LB, Kauffman CA. Voriconazole: a new triazole antifungal agent. Clin Infect Dis 2003;36:630-7.

21. Panackal AA, Williamson PR. Fungal Infections of the Central Nervous System. Continuum (Minneap Minn) 2015;21:1662-78.

22. Kojic EM, Darouiche RO. Candida infections of medical devices. Clin Microbiol Rev 2004;17:255-67.

23. Takase K, Yoshida T, Nakamura T, et al. Cryptococcal Meningitis in a Patient with Breast Cancer Receiving Everolimus: A Case of Successful Treatment with Continuous Cerebrospinal Fluid Drainage. Brain Nerve 2018;70:1301-5.

24. Glick JA, Graham RS, Voils SA. Candida meningitis post Gliadel wafer placement successfully treated with intrathecal and intravenous amphotericin B. Ann Pharmacother 2010;44:215-8.

25. Toprak D, Öcal Demir S, Kadayifci EK, et al. Recurrent Candida albicans Ventriculitis Treated with Intraventricular Liposomal Amphotericin B. Case Rep Infect Dis 2015;2015:340725.

(English Language Editor: A. Kassem) 